\title{
A Combined Raman-Fluorescence Spectroscopic Probe for Tissue Diagnostics Applications
}

\author{
Riccardo Cicchi ${ }^{a, b}$ Alessandro Cosci,${ }^{b}$ Susanna Rossari ${ }^{c}$ Alessandro Sturiale, ${ }^{d}$ Flavio Giordano, ${ }^{e}$ \\ Vincenzo De Giorgi, ${ }^{c}$ Vincenza Maio, ${ }^{f}$ Daniela Massi,${ }^{f}$ Gabriella Nesi,${ }^{f}$ Anna Maria Buccoliero, ${ }^{f}$ \\ Francesco Tonelli, ${ }^{d}$ Renzo Guerrini, ${ }^{e}$ Nicola Pimpinelli, ${ }^{c}$ and Francesco Saverio Pavone ${ }^{b, g}$ \\ ${ }^{a}$ National Institute of Optics, National Research Council (INO-CNR), Largo Enrico Fermi 6 - \\ 50125, Florence, Italy; \\ ${ }^{\mathrm{b}}$ European Laboratory for Non-Linear Spectroscopy (LENS), University of Florence, Via Nello \\ Carrara, 1 - 50019, Sesto Fiorentino, Italy; \\ ${ }^{\mathrm{c}}$ Division of Clinical, Preventive and Oncology Dermatology, Department of Critical Care Medicine \\ and Surgery, University of Florence, Piazza Indipendenza 11 - 50129, Florence, Italy; \\ ${ }^{\mathrm{d}}$ Department of Clinical Physiopathology, Surgical Unit, University of Florence, Florence, Viale \\ Giovanni Battista Morgagni 85 - 50134, Florence, Italy; \\ e Division of Neurosurgery, Department of Neuroscience I, "Anna Meyer" Pediatric Hospital, Viale \\ Gaetano Pieraccini 24 - 50141, Florence, Italy; \\ ${ }^{\mathrm{f}}$ Division of Pathology, Department of Critical Care Medicine and Surgery, University of \\ Florence, Viale Giovanni Battista Morgagni 85 - 50134, Florence, Italy; \\ ${ }^{\mathrm{g}}$ Department of Physics, University of Florence, Via Giovanni Sansone 1 - 50019, Sesto Fiorentino, \\ Italy; \\ Corresponding author E-mail address: riccardo.cicchi@ino.it
}

\begin{abstract}
We designed and developed two different optical fibre probes for combined Raman and fluorescence spectroscopic measurements on human tissues. The experimental setup combines fluorescence spectroscopy and Raman spectroscopy in a multimodal approach. Two laser diodes, respectively emitting in the UV (378 nm) and in the visible $(445 \mathrm{~nm})$, were used for fluorescence spectroscopy. An additional laser diode emitting in the NIR (785 nm) was used for Raman spectroscopy. Laser light was delivered to the tissue under examination through a multimode optical fibre located in the centre of the fibre bundle probe. The surrounding 24 optical fibres were used for collection of the signal of interest and for delivering light to a common detection unit. Both fluorescence and Raman spectra were acquired on a cooled CCD camera, connected to a spectrograph. The device was successfully used for diagnosing melanocytic lesions in a good agreement with common routine histology. Additional measurements were performed on other human tissue samples, such as colon tissue and brain tissue in order to test the capability of the device for diagnosing a broader range of tissue lesions and malignancies. The system has the potential to improve diagnostic capabilities on a broad range of tissues and to be used for endoscopic inspections in the near future.
\end{abstract}

Keywords: (300.6280) Spectroscopy, fluorescence and luminescence; (300.6450) Spectroscopy, Raman; (170.6935) Tissue characterization;

\section{INTRODUCTION}

Skin cancer is one of the most common and worldwide disease. Even if Malignant Melanoma (MM) represents only the $2 \%$ of all skin cancer, MM is characterized by its high aggressiveness and death incidence [1]. Currently MM is diagnosed by visual inspection using while light dermoscopy. Suspected lesions, according to the ABCDE law [2, 3], are then excised and undergo to histopathological examination. This method is strongly dependent on the dermatologist experience, is invasive and time-consuming since the histopathological response usually requires several days. Therefore

Clinical and Biomedical Spectroscopy and Imaging III, edited by Volker Deckert, Nirmala Ramanujam,

Proc. of OSA Biomedical Optics-Vol. 8798, 87980C · (c) 2013 OSA-SPIE

CCC code: $1605-7422 / 13 / \$ 18 \cdot$ doi: $10.1117 / 12.2031370$ 
in these last two decades new techniques, based on light spectroscopy, have been proposed for improving diagnostic accuracy. The recent introduction of optical and spectroscopic techniques in the clinical settings contributed to the achievement of a more accurate tissue diagnostics. Such techniques are usually referred as "optical biopsy". "Optical biopsy" uses the interaction of light with the tissue in order to have an indication of its chemical composition. Since light penetration in skin is in the order of some millimeter "optical biopsy" does not require tissue excision. Most of these techniques are based on tissue auto-fluorescence or on spontaneous Raman scattering. Laser-induced auto-fluorescence of tissue takes advantage of the intrinsic fluorescence produced by endogenous molecules and has been already demonstrated as a possible method for diagnosing pathological tissue conditions, such as dysplastic or neoplastic. Raman spectroscopy takes advantage of inelastic scattering process in which non-resonant photons contribute to the excitation of vibrational molecular modes. The measurement of the energy lost in the process allows to measure a vibrational spectrum, characteristic of the molecule involved in the process. Fluorescence and Raman spectroscopy have been demonstrated to be effective in detecting skin cancer $[4,5,6,7,8]$. The former uses radiation in the ultraviolet (UV) and visible light in order to excite tissue endogenous molecules without any need of contrast agent [9].

One important application of fluorescence spectroscopy in cancer diagnosis is the possibility to score the tissue metabolism through the Warburg effect $[10,11,12]$. Cell energy production can occur by two different metabolic pathway: oxidative phosphorylation and glycolysis. The former, typical of healthy tissues, requires oxygen and involves both nicotinamide adenine dinucleotide (NADH) and flavin adenine dinucleotide (FAD), two important molecules in cell metabolism. Tumor tissues have a higher metabolism and usually oxygen supplied by blood vessels is not enough. Therefore they switch the metabolic pathway to glycolysis, which is not oxygen dependent and involves only NADH. By the spectroscopic point of view the two molecules presents two distinct excitation and emission spectra. NADH is usually excited at $370 \mathrm{~nm}$, and emits mainly at $460 \mathrm{~nm}$. Whilst FAD is excited at $445 \mathrm{~nm}$ and has an emission peak at $510 \mathrm{~nm}$. Hence, exciting the tissue at the two different wavelengths and collecting the respective fluorescence is it possible to have a measurement of the balance between NADH and FAD, and therefore a measurement of the metabolic pathway of cells. Besides skin cancer this technique has been successfully employed for enhancing the diagnosis of tumor in Barret's esophagus [13], bladder [14], lung [15]. Fluorescence spectroscopy was also used for monitoring organ transplantation [16] and medical treatment [17]. Excitation wavelength in the IR range were found to be particularly useful in MM diagnosis [18, 19], since they are selectively exciting melanin.

Raman spectroscopy is another spectroscopic technique widely used in tissue diagnosis. It uses inelastic scattering of IR radiation with the molecules. Since the energy levels of molecules are quantized, the energy lost or acquired by the incident photon is quantized as well. Therefore, Raman spectroscopy gives a measurement of the vibrational energy separation of the molecule investigated. While fluorescence spectrum of the tissue is an overlapping of the single fluorescence emission of the composing molecules, Raman spectrum is characteristic of each molecule, hence, has an higher specificity. Cancer diagnosis by Raman spectroscopy has already been demonstrated in colon [20], brain [21] and gastrointestinal tract [22]. Raman spectroscopy was found to be effective in removing fluorescent false positive in aminolevulinic acid (ALA) fluorescence cystoscopy of bladder [23]. In MM diagnosis it was used in conjunction of principal components analysis (PCA) and neural network leading to a $85 \%$ sensitivity and $99 \%$ specificity [7]. Further improvements for in vivo application of spectroscopic techniques were achieved by the introduction of fiber probes [24, 25]. Such probes use optical fiber for deliver the excitation light to the tissue and collecting the fluorescence/Raman signal. By means of optical fiber the probe results to be highly flexible and can be extremely compact and small, in order to be used in the service channel of standard commercial endoscope. One of the main concerns in Raman fiber probes is the own fluorescence and Raman signal produced by the fibers that can overwhelm the signal out from the tissue [26, 27]. Therefore, a narrow bandpass filter, center around the laser line, should be placed in front of the delivering fiber at the tip of the bundle. Another longpass filter should be used instead in front of the collecting fibers, in order to block the Rayleigh back-scattered laser light. Unfortunately at the moment it not possible to use such filtered fiber bundle to accomplish fluorescence spectroscopy, since the filter used are not transparent to the wavelength used usually in the VIS-UV range. Therefore our system uses two different probes, one for fluorescence and the other for Raman spectroscopy.

In this study we designed and developed two different optical fibre probes for combined Raman and fluorescence spectroscopic measurements on human tissues. The two devices were successfully used for diagnosing melanocytic lesions in a good agreement with common routine histology, and additionally tested on colon and brain tissues. 


\section{MATERIALS AND METHODS}

\subsection{Fluorescence excitation}

Fluorescence spectroscopy was performed using two laser diodes as excitation sources (TEC 042, Sacher Lasertechnik, Maburg, Germany). The two sources provide excitation in the UV $(378 \mathrm{~nm})$ and in the visible spectral range $(445 \mathrm{~nm})$, as shown in Figure 1. The two wavelengths were chosen in order to respectively excite the main tissue intrinsic fluorophores, NADH and FAD. The two laser beams are superimposed on the same optical path by means of a dichroic beam splitter (FF409-Di02-25x36, Semrock, Rochster, NY, US), then optically coupled to the central fibre of a fibre bundle by means of fibre coupler equipped with an SMA connector (Ely-Prizmatix, Modiin Ilite, Israel). Unwanted back reflected light from the fibre coupler were prevented by placing a polarizer cube (GTH10M, Thorlabs, Newton, NJ, US), followed by a quarter-wavelength plate (WPA1212-L/4-375-445, Casix, Fozhou Fujian, China), in the optical path. Laser light transmission through the polarizer was maximized by inserting, and properly orienting, two half-wavelength plates (WPO-12.7CQ-0-2-442 and WPO-12.7-0-2-355, Lambda, Costa Mesa, CA, US) in front of each laser source.

\subsection{Raman excitation}

Although the strength of the spontaneous Raman signal increases when decreasing excitation wavelength [28], the choice of the excitation wavelength for Raman spectroscopy is particularly critical when the specimen is a biological tissue. Theoretically, UV and VIS wavelengths are more suitable because of a larger cross-section for spontaneous Raman scattering. On the other hand, biological tissues provide a fluorescence signal under UV/VIS illumination that could mask the Raman signal of interest. For this reason, in applications involving tissue spectroscopy, wavelengths in the range of NIR (between $780 \mathrm{~nm}$ and $830 \mathrm{~nm}$ ) are more preferable for minimizing the fluorescence contribution. In our set-up we used a diode laser emitting at $785 \mathrm{~nm}$ (FC-785-350-MM2-PC-1-0-RM, RGBLase, Fremont, CA, US). The laser was equipped with an $\mathrm{FC} / \mathrm{PC}$ connector in order to be directly coupled to the central fibre of a fibre bundle.

\subsection{Fluorescence fibre probe}

The fluorescence fibre bundle probe (EMVision LCC, Laxahatchee, FL, US) was made by 25 multimode optical fibres (100 microns core diameter, LOW-OH, NA 0.22). The central fibre was used for delivering excitation light to the tissue under investigation (Figure 1). The other fibres were used for collecting fluorescence light by placing them around the central fibre in a honeycomb geometry. A bifurcation in the fibre bundle allowed connecting the central fibre to the fibre coupler and the collecting fibres to the detection system. At the proximal end collecting fibres were arranged in a line in order to maximize light transmission through the entrance slit of the detection system. The linear arrangement allowed inserting an MTP optical fibre connector for rapidly switching among different bundles.

\subsection{Raman fibre probe}

The Raman fibre bundle probe (EMVision LCC, Laxahatchee, FL, US) was made as well by 25 multimode optical fibres (100 microns core diameter, LOW-OH, NA 0.22 ). The central fibre was directly plugged to the laser source through an $\mathrm{FC} / \mathrm{PC}$ connector and used for delivering excitation light to the tissue under investigation (Figure 1). The other fibres were used for collecting Raman scattered light by placing them around the central fibre in a honeycomb geometry. In order to remove unwanted fluorescence and Raman signal generated inside the delivering fibre, a custom-made bandpass filter $(10 \mathrm{~nm}$ bandwidth, central wavelength $785 \mathrm{~nm}$ ) was placed at the distal end of the probe, in front of the excitation fibre. Another ring-shaped long pass filter placed in front of the collecting fibres prevented Rayleigh back-scattered photons from entering the detection fibres. Both filters were glued on the tip of the fibre bundle probe. A bifurcation in the fibre bundle, similar to that one of the fluorescence probe, allowed connecting the central fibre to the fibre coupler and the collecting fibres to the detection system. At the proximal end collecting fibres were arranged in a line in order to maximize light transmission through the entrance slit of the detection system. The linear arrangement allowed to insert an MTP optical fibre connector for rapidly switching between fluorescence and Raman measurements. 
Light Delivering

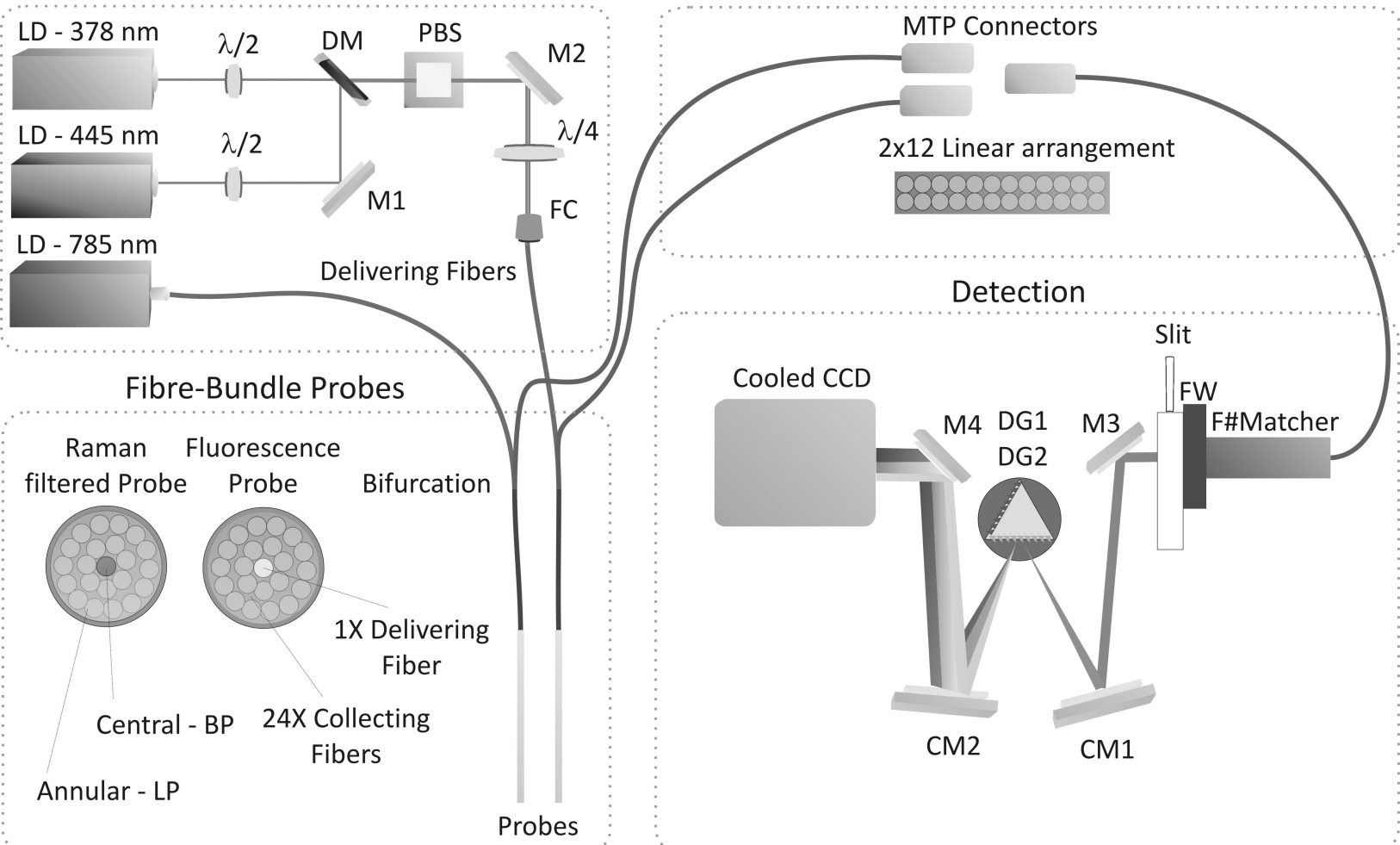

Figure 1. Schematic view of the experimental setup divided in blocks: Light delivering (top left), Fibre-bundle probes (down left), Bundle connector (top right), Detection (down right). A magnified sketch of the optical fibre arrangement at the fibre tips and at the bundle MTP connector are shown in the Fibre-Bundle Probes and in the Bundle Connector blocks, respectively. (LD: laser diode; $\lambda / 2$ : half waveplate; DM: dichroic mirror; M: mirror; PBS: polarizing beamsplitter; 1/4: quarter waveplate; FC: fibre coupler; BP: bandpass; LP: longpass; FW: filter wheel; CM: curved mirror; DG: diffraction grating). Figure derived from Figure 1 in [29].

\subsection{Detection system}

The detection system was based on a 1/4m monochromator (Oriel Cornerstone, Newport, Irvine, CA, US) equipped with two different gratings, one for fluorescence spectroscopy (600 lines $/ \mathrm{mm}$, blaze wavelength $500 \mathrm{~nm}$ ) and one for Raman spectroscopy (1200 lines $/ \mathrm{mm}$, blaze wavelength $750 \mathrm{~nm}$ ). A cooled CCD array camera (iDus 420 BRDD, Andor, Belfast, Northern Ireland, UK) was placed at the output port of the monochromator. The two fibre bundles were connected to the detection system by means of a fibre bundle branch (EMVision LCC, Laxahatchee, FL, US) with 24 optical fibers (100 microns core diameter, LOW-OH, NA 0.22), arranged in two lines. An F\# matcher was inserted between the branch and the monochromator in order to match the numerical aperture of the fibres with the F\# of the monochromator. A motorized filter wheel was placed between the F\# matcher and the monochromator. Three different long pass filters were used in order to prevent excitation light from entering the detection system. The filters were changed when changing excitation wavelength. For fluorescence spectroscopy at $378 \mathrm{~nm}$ excitation we used a long pass at $400 \mathrm{~nm}$ (Longpass $400 \mathrm{~nm}$, Melles Griot, Albuquerque, NM, US). For fluorescence spectroscopy at $445 \mathrm{~nm}$ excitation we used a long pass at $458 \mathrm{~nm}$ (LP02-458RS-25, Semrock, Rochester, NY, US). For Raman spectroscopy we used a long pass at $785 \mathrm{~nm}$ (LP02-785RE-25, Semrock, Rochester, NY, US).

\subsection{Samples and measurements}

Fresh biopsies of tissues were excised and immediately transported in our lab for spectroscopic measurements. Acquisition times were in the order of $0.2 \mathrm{~s}-2 \mathrm{~s}$ for fluorescence spectroscopy, and $10 \mathrm{~s}$ for Raman spectroscopy. Inspection requires less than 2 minutes for each sample. After the measurements samples were rinsed in formalin for the 
subsequent histopathological examination. The relevant clinical data of the examined skin samples are summarized in Table 1.

\begin{tabular}{|c|c|c|c|c|c|}
\hline Age & Gender & Body site & Lesion Size & Clinical Diagnosis & Histological Diagnosis \\
\hline 19 & F & Arm & $11 \times 6 \mathrm{~mm}^{2}$ & Nevus & Nevus \\
\hline 39 & F & Arm & $5 \times 4 \mathrm{~mm}^{2}$ & Melanoma & Melanoma in situ \\
\hline 55 & F & Trunk & $8 \times 4 \mathrm{~mm}^{2}$ & Melanoma & Melanoma in situ \\
\hline 34 & M & Chest & $5 \times 5 \mathrm{~mm}^{2}$ & Nevus & Nevus \\
\hline 67 & M & Back & $6 \times 6 \mathrm{~mm}^{2}$ & Nevus & Melanoma \\
\hline 85 & M & Back & $10 \times 20 \mathrm{~mm}^{2}$ & Melanoma & Melanoma \\
\hline 45 & F & Chest & $10 \times 5 \mathrm{~mm}^{2}$ & Melanoma & Nevus \\
\hline 61 & F & Back & $9 \times 5 \mathrm{~mm}^{2}$ & Melanoma & Melanoma \\
\hline 37 & F & Back & $9 \times 4 \mathrm{~mm}^{2}$ & Nevus & Nevus \\
\hline 21 & M & Forearm & $7 \times 4 \mathrm{~mm}^{2}$ & Nevus & Nevus \\
\hline 50 & F & Back & $2 \times 3 \mathrm{~mm}^{2}$ & Reed Nevus & Reed Nevus \\
\hline 37 & F & Back & $11 \times 9 \mathrm{~mm}^{2}$ & Melanoma & Melanoma \\
\hline 40 & F & Trunk & $20 \times 8 \mathrm{~mm}^{2}$ & Nevus & Nevus \\
\hline 71 & M & Scalp & $15 \times 10 \mathrm{~mm}^{2}$ & Melanoma & Melanoma \\
\hline 55 & F & Thigh & $9 \times 4 \mathrm{~mm}^{2}$ & Melanoma & Melanoma \\
\hline 46 & M & Trunk & $6 \times 9 \mathrm{~mm}^{2}$ & Nevus & Nevus \\
\hline 64 & F & Foot & $11 \times 8 \mathrm{~mm}^{2}$ & Melanoma & Melanoma \\
\hline 63 & M & Arm & $15 \times 10 \mathrm{~mm}^{2}$ & Melanoma & Melanoma \\
\hline 18 & M & Back & $15 \times 8 \mathrm{~mm}^{2}$ & Nevus & Nevus \\
\hline 21 & M & Abdomen & $6 \times 6 \mathrm{~mm}^{2}$ & Nevus & Nevus \\
\hline
\end{tabular}

Table 1. Relevant clinical data of the examined skin samples.

\section{RESULTS AND DISCUSSION}

The diagnostic capabilities of the method in discriminating between Malignant Melanoma (MM) and Melanocyctic Naevus (MN) were evaluated by means of a scoring algorithm, designed by taking a linear combination of the first PCA coefficients obtained from various measurements $(378 \mathrm{~nm}$ fluorescence $-445 \mathrm{~nm}$ fluorescence $-785 \mathrm{~nm}$ fluorescence Raman). In order to separately evaluate the diagnostic capabilities of VIS and NIR spectroscopy, three different scoring algorithms were developed as follows:

$$
\begin{aligned}
& \text { Score }(\text { VIS })=\operatorname{PCA1}(378 \mathrm{~nm})+\operatorname{PCA1}(445 \mathrm{~nm}) \\
& \text { Score }(\mathrm{NIR})=\text { PCA1 }(785 \mathrm{~nm}) \text { - PCA1 }(\text { Raman }) \\
& \text { Score }(\text { Combined })=\text { Score }(\mathrm{VIS})+\text { Score }(\mathrm{NIR})
\end{aligned}
$$

where (1) represents the scoring algorithm used for evaluating the diagnostic capability of VIS spectroscopy, (2) represents the scoring algorithm for evaluating diagnostic capability of NIR spectroscopy, and (3) is the scoring algorithm for the combination of the two approaches. A classifier was defined for each scoring algorithm. The average score for all MM samples and for all MN samples was calculated. Half of the sum of the two average scores were taken as thresholds. These thresholds were used as classifiers. In particular, a sample is classified as MM or MN depending if the Score is higher or lower than the threshold.

The diagnostic capabilities in terms of sensitivity and specificity were evaluated on the basis of the scores of above, using a cross-validation method employing a leave-one-out approach. The results in terms of sensitivity and specificity are summarized in the table below: 


\begin{tabular}{|c|c|c|c|}
\hline $\begin{array}{c}\text { Scoring } \\
\text { algorithm }\end{array}$ & VIS & NIR & Combined \\
\hline Sensitivity & $78 \%$ & $56 \%$ & $89 \%$ \\
\hline Specificity & $89 \%$ & $89 \%$ & $100 \%$ \\
\hline
\end{tabular}

Table 2. Summary of the Diagnostic capabilities.

The comparison of single techniques with respect to the combined techniques demonstrated a significant enhancement of the diagnostic capabilities in terms of both sensitivity and specificity arising from the combination of the two techniques. In particular, it is worth noting that a $89 \%$ sensitivity and a $100 \%$ specificity can be obtained.

\section{CONCLUSION}

In this work we presented a new spectroscopic system based on fibre probes for combined fluorescence and Raman spectroscopy. The probes have been tested on freshly excised biopsies of skin, demonstrating the capability to discriminate between $\mathrm{MN}$ and $\mathrm{MM}$ in a good agreement with "gold-standard" histology. The combination of fluorescence and Raman spectroscopy provided a more detailed classification of lesions. In particular, a cross-check of the two techniques can provide a more accurate diagnosis in terms of both sensitivity and specificity. As a future perspective of this work, it is planned to enlarge the number of examined samples in order to obtain a more relevant statistics as well as to perform a more challenging in vivo study. The extremely reduced size of the device may allow its use also in endoscopy in the next future. With this study it has been demonstrated that the system can potentially contribute to improve clinical diagnostic capabilities and hence reduce the number of unnecessary biopsies in dermatology. In addition, the combination of fluorescence and Raman spectroscopy provided also a more detailed classification of colon and brain lesions. Our system can potentially contribute to improve clinical diagnostic capabilities on a broad range of tissues.

\section{ACKNOWLEDGEMENT}

The research leading to these results has received funding from the European Union Seventh Framework Programme (FP7/2007-2013) under grant agreements number 228334 and 284464, and from the Italian Ministry for Education, University and Research in the framework of the Flagship Project NANOMAX. Furthermore, financial support by Ente Cassa di Risparmio di Firenze (private foundation) is acknowledged.

\section{REFERENCES}

[1] http://www.cancer.org/Research/CancerFactsFigures/CancerFactsFigures/ACSPC-029771.

[2] R R. Friedman, D. Rigel, and A. Kopf. Early detection of malignant melanoma: the role of physician examination and self-examination of the skin. CA Cancer J Clin, 35(3):130-151, 1985.

[3] http://www.melanomafoundation.org/prevention/abcd.htm.

[4] L. Brancaleon, A. J. Durkin, J. H. Tu, G. Menaker, J. D. Fallon, and N. Kollias. In vivo fluorescence spectroscopy of nonmelanoma skin cancer. Photochemistry and Photobiology, 73:178-183, 2001.

[5] R. Cicchi, D. Massi, S. Sestini, P. Carli, V. De Giorgi, T. Lotti, and F. S. Pavone. Multidimensional non-linear laser imaging of basal cell carcinoma. Optics Express, 15(16):10135-10148, Aug 2007.

[6] S. Bandeira, S. I. Dunshee de A. Oliveira, B. Renata, O. A. Fernandes, F. L.Masako, M. H. Silva, and M. A. A. Cartaxo. FT-Raman spectroscopy for the differentiation between cutaneous melanoma and pigmented nevus. Acta Cirurgica Brasileira, 25:351-356, 082010.

[7] M. Gniadecka, P. A. Philipsen, S. Sigurdsson, S. Wessel, O. F. Nielsen, D. H. Christensen, J. Hercogova, K. Rossen, H. K. Thomsen, R. Gniadecki, L. K. Hansen, and H. C. Wulf. Melanoma diagnosis by Raman spectroscopy 
and neural networks: Structure alterations in proteins and lipids in intact cancer tissue. J Investig Dermatol, (122):443449, 2004.

[8] Masoud Panjehpour, Clark E. Julius, Mary N. Phan, Tuan Vo-Dinh, and Suzanne Overholt. Laser-induced fluorescence spectroscopy for in vivo diagnosis of non-melanoma skin cancers. Lasers in Surgery and Medicine, 31(5):367-373, 2002.

[9] G. A. Wagnieres, W. M. Star, and B. C. Wilson. In vivo fluorescence spectroscopy and imaging for oncological applications. Photochemistry and Photobiology, 68:1751-1097, 1998.

[10] O. Warburg. The metabolism of tumor. Constabel, London, 1930.

[11] M. C. Skala, K. M. Riching, A. Gendron-Fitzpatrick, J. Eickhoff, K. W. Eliceiri, J. G. White, and N. Ramanujam. In vivo multiphoton microscopy of NADH and FAD redox states, fluorescence lifetimes, and cellular morphology in precancerous epithelia. Proceedings of the National Academy of Sciences, 104(49):19494-19499, 2007.

[12] N. Ramanujam, R. Richards-Kortum, S. Thomsen, A. Mahadevan-Jansen, M. Follen, and B. Chance. Low temperature fluorescence imaging of freeze trapped human cervical tissues. Optics Express, 8(6):335-343, 2001.

[13] T.J. Pfefer, D. Y. Paithankar, J. M. Poneros, K.T. Schomacker, and N. S. Nishoka. Temporally and spectrally resolved fluorescence spectroscopy for the detection of high grade dysplasia in barrett's esophagus. Lasers in Surgery and Medicine, 32:10-16, 2003.

[14] W. Zheng, W. Lau, C. Cheng, K. C. Soo, and M. Olivo. Optimal excitation-emission wavelengths for autofluorescence diagnosis of bladder tumors. Int. J. Cancer, 104:477-481, 2003.

[15] D. Httenberger, T. Gabrecht, G. Wagnires, B. Weber, A. Linder, H.J. Foth, and L. Freitag. Autofluorescence detection of tumors in the human lung spectroscopical measurements in situ, in an in vivo model and in vitro. Photodiagnosis and Photodynamic Therapy, 5:139-147, 2008.

[16] O. Castro e Silva, A.K. Sankarankutty, R.B. Correa, J. Ferreira, J.D. Vollet Filho, C. Kurachi, and V.S. Bagnato. Autfluorescence spectroscopy in liver transplantation: Preliminary results from a pilot clinical study. Transplantation Proceedings, 40:722-725, 2008.

[17] J. D. Vollet-Filho, P. F. C. Menezes, L. T. Moriyama, C. Grecco, C. Sibata, R. R. Allison, O. Castro e Silva, and V. S. Bagnato. Possibility for a full optical determination of photodynamic therapy outcome. Journal of Applied Physics, 105(10):102038-102038-7, may 2009.

[18] Z. Huang, H. Zeng, I. Hamzavi, A. Alajlan, E. Tan, D. I. McLean, and H. Lui. Cutaneous melanin exhibiting fluorescence emission under near-infrared light excitation. Journal of Biomedical Optics, 11(3):034010, 2006.

[19] D. Leupold, M. Scholz, G. Stankovic, J. Reda, S Buder, R. Eichhorn, G. Wessler, M. St"cker, K. Hoffmann, J. Bauer, and C. Garbe. The step-wise two-photon excited melanin fluorescence is a unique diagnostic tool for the detection of malignant transformation in melanocytes. Pigment Cell \& Melanoma Research, 24(3):438-445, 2011.

[20] A. Molckovsky, L. M. Wong Kee Song, M. G. Shim, N. E. Marcon, and B. C. Wilson. Diagnostic potential of nearinfrared Raman spectroscopy in the colon: Differentiating adenomatous from hyperplastic polyps. Gastrointestinal Endoscopy, 57(3):396-402, 2003.

[21] A. Mahadevan-Jansen, M. F. Mitchell, N. Ramanujam, U. Utzinger, and R. Richards-Kortum. Development of a fiber optic probe to measure nir Raman spectra of cervical tissue in vivo. Photochemistry and Photobiology, 68(3):427-431, 1998.

[22] Martin G. Shim, Louis-Michel Wong Kee Song, Norman E. Marcon, and Brian C. Wilson. In vivo near-infrared Raman spectroscopy: Demonstration of feasibility during clinical gastrointestinal endoscopy. Photochemistry and Photobiology, 72(1):146-150, 2000.

[23] S. Denzinger, M. Burger, B. Walter, R. Knuechel, W. Roessler, W. F. Wieland, and T. Filbeck. Clinically relevant reduction in risk of recurrence of superficial bladder cancer using 5-aminolevulinic acid-induced fluorescence diagnosis: 8-year results of prospective randomized study. Urology, 69(4):675-679, 2007. 
[24] U. Utzinger and R. R. Richards-Kortum. Fiber optic probes for biomedical optical spectroscopy. J. Biomed. Opt., 8(1):121-147, 2003.

[25] N. D. Magee, J. S. Villaumie, E. T. Marple, M. Ennis, J. S. Elborn, and J. J. McGarvey. Ex vivo diagnosis of lung cancer using a Raman miniprobe. The Journal of Physical Chemistry B, 113(23):8137-8141, 2009. PMID: 19453143.

[26] M. G. Shim, B. C. Wilson, E. Marple, and M. Wach. Study of fiber-optic probes for in vivo medical Raman spectroscopy. Appl. Spectrosc., 53(6):619-627, Jun 1999.

[27] J. T. Motz, M. Hunter, L. H. Galindo, J. A. Gardecki, J. R. Kramer, R. R. Dasari, and M. S. Feld. Optical fiber probe for biomedical Raman spectroscopy. Appl. Opt., 43(3):542-554, Jan 2004.

[28] M. Diem. Introduction to modern vibrational spectroscopy. Wiley, 1993.

[29] R. Cicchi, A. Cosci, S. Rossari, D. Kapsokalyvas, E. Baria, V. Maio, D. Massi, V. De Giorgi, N. Pimpinelli, and F. S. Pavone. Combined fluorescence-Raman spectroscopic setup for the diagnosis of melanocytic lesions. Journal of Biophotonics, DOI 10.1002/jbio.201200230 (2013) 